\title{
Epigenetic Alterations in Inflammatory Bowel Disease and Cancer
}

\author{
Joo Mi Yi ${ }^{1}$, Tae Oh $\mathrm{Kim}^{2}$ \\ Research Institute, Dongnam Institute of Radiological \& Medical Sciences (DIRAMS)', Department of Internal Medicine, Haeundae Paik \\ Hospital, Inje University College of Medicine $e^{2}$, Busan, Korea
}

Overwhelming evidences supports the idea that inflammatory bowel disease (IBD) is caused by a complex interplay between genetic alterations of multiple genes and an aberrant interaction with environmental factors. There is growing evidence that epigenetic factors can play a significant part in the pathogenesis of IBD. Significant effort has been invested in uncovering genetic and epigenetic factors, which may increase the risk of IBD, but progress has been slow, and few IBD-specific factors have been detected so far. It has been known for decades that DNA methylation is the most well studied epigenetic modification, and analysis of DNA methylation is leading to a new generation of cancer biomarkers. Therefore, in this review, we summarize the role of DNA methylation alteration in IBD pathogenesis, and discuss specific genes or genetic loci using recent molecular technology advances. Here, we suggest that DNA methylation should be studied in depth to understand the molecular pathways of IBD pathogenesis, and discuss epigenetic studies of IBD that may have a significant impact on the field of IBD research.

(Intest Res 2015;13:112-121)

Key Words: Inflammatory bowel diseases; Neoplasms; Epigenomics; DNA methylation

\section{INTRODUCTION}

Tumorigenesis is a multistep process, including initiation, promotion, and progression, and a multifactorial pathology characterized by the accumulation of a multitude of alterations, including genetic, cytogenetic, and epigenetic changes. ${ }^{1}$ To completely construct an organism, classical genetic processes are not sufficient. For proper development and cell functioning, epigenetic phenomena, controlling gene expression, are absolutely required. The term "epigenetic" refers to a heritable change in the pattern of gene expression

Received December 24, 2014. Revised January 14, 2015.

Accepted January 14, 2015.

Correspondence to Tae Oh Kim, Department of Internal Medicine,

Haeundae Paik Hospital, Inje University College of Medicine, 875 Haeun-

daero, Haeudae-gu, Busan 612-896, Korea. Tel: +82-51-797-0200, Fax:

+82-51-797-0298, E-mail: kto0440@paik.ac.kr

Financial support: This study was supported by the National REtD program (50596-2015) through the Dongnam Institute of Radiological \& Medical Sciences (DIRAMS) funded by the Ministry of Science, ICT and Future Planning (MSIP), Korean government. Conflict of interest: None. that is mediated by mechanisms other than alterations in the primary nucleotide sequence of a gene. ${ }^{2,3}$ As evidence for genetic changes in cancer cells has increased since the 1980s, interest in the contribution of epigenetic changes to neoplasia has waned. The situation has changed dramatically in more recent years, however, because of convincing evidence for the abnormal silencing of genes in cancer cells. ${ }^{4,5} \mathrm{It}$ has been known that epigenetic alteration is one of the most important gene regulatory mechanisms. Unlike genetic alterations, epigenetic events are not changes in gene function that occur in conjunction with DNA sequence changes.

Epigenetic regulation of gene expression is mediated by mechanisms such as DNA methylation, histone modification, and positioning of nucleosome along DNA. The interplay between epigenetic components guarantees a proper balance between transcriptional activity and repression by changing chromatin architecture. Thus, regulation of the packaging of DNA ensures maintenance of correct chromosome replication, gene expression, and stable gene silencing. ${ }^{6}$ DNA methylation is one of the most intensely studied

๑ Copyright 2015. Korean Association for the Study of Intestinal Diseases. All rights reserved.

This is an Open Access article distributed under the terms of the Creative Commons Attribution Non-Commercial License (http://creativecommons.org/licenses/by-nc/3.0)

which permits unrestricted non-commercial use, distribution, and reproduction in any medium, provided the original work is properly cited. 
epigenetic modifications in mammals and has an important impact on normal cell physiology. As this DNA modification seems to be a critical player in transcriptional regulation, it is not surprising that defects in this mechanism may lead to various diseases, including cancer. ${ }^{7}$ Recently, epigenetic studies have been conducted in many different fields of biology, and particularly in the cancer field.

\section{THE MOLECULAR BASIS OF DNA METHYLATION}

Although only four bases -adenine, guanine, cytosine, and thymidine- compose the primary sequence of DNA, covalent modification of postreplicative DNA, that is DNA that has replicated itself in a dividing cell, that produces a "fifth base". A reaction using S-adenosyl-methionine as a methyl donor and catalyzed by enzymes called DNA methyltransferases (DNMTs) adds a methyl group to the cytosine ring to
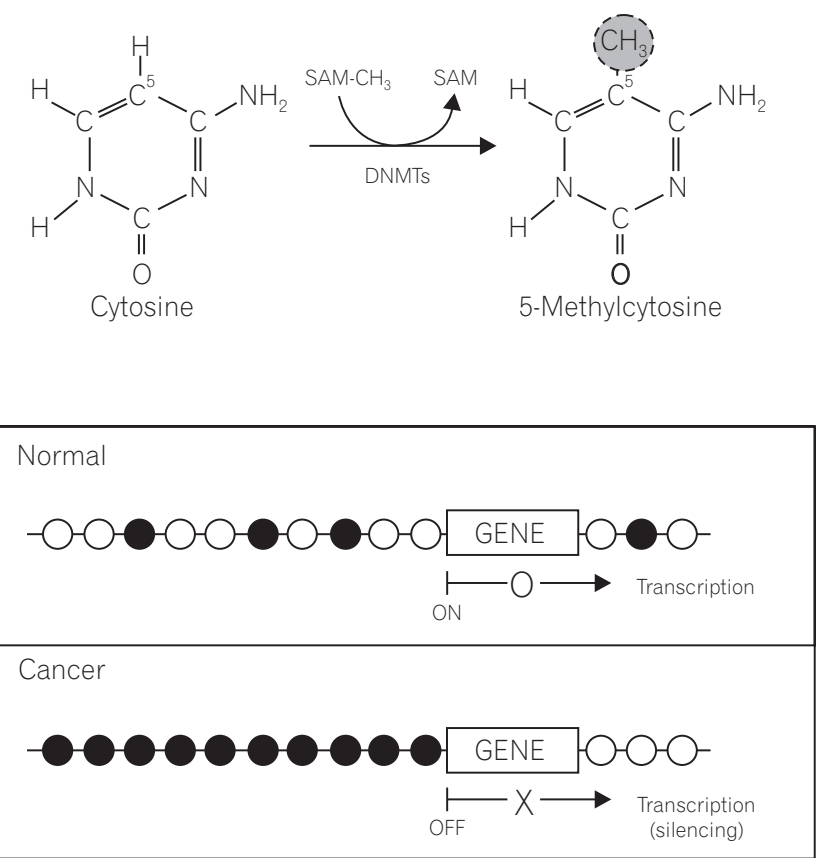

Fig. 1. Methylation patterns between normal cells and tumor cells. (A) Conversion of cytosine to 5-methylcytosine by DNA methyltransferase (DNMT). DNMT catalyzes the transfer of a methyl group (CH3) from Sadenosylmethionine (SAM) to the 5-carbon position of cytosine. (B) Methylation patterns between normal cells and tumor cells. In normal cells, cytosine-phospho-guanine $(\mathrm{CpG})$ sites are globally methylated which means most $\mathrm{CpG}$ sites outside $\mathrm{CpG}$ islands are methylated (black circles), whereas most $\mathrm{CpG}$ island sites in gene expression promoters are unmethylated (white circles). Therefore, unmethylation status of $\mathrm{CpG}$ islands in gene promoters permits active gene expression. In cancer cells, $\mathrm{CpG}$ islands in gene promoter regions become abnormally methylated and this causes transcriptional silencing of genes. Circles indicate CpG dinucleotides. form methyl cytosine (Fig. 1A).

In humans and other mammals, this modification is imposed only on cytosines that precede a guanosine in the DNA sequence (the cytosine-phospho-guanine [CpG] dinucleotide). The overall frequency of CpGs in the genome is substantially less than what would be mathematically predicted. The distribution in the genome of $\mathrm{CpG}$ dinucleotides on which DNA methylation occurs is unusually asymmetric. In contrast to the relative small portion of CpGs in the genome as a whole, these dinucleotides can be clustered in small stretches of DNA termed "CpG islands". These regions are often associated with sites where the transcription of DNA into RNA begins, which can be the promoter region of genes.

The methylation reaction of cytosines is mediated by a class of enzymes called DNMTs that catalyze the transfer of the methyl group from s-adenosyl-methionine onto cytosine. Five members of the DNMT family have been identified in mammals: DNMT1, DNMT2, DNMT3a, DNMT3b, and DNMT3L. However, as far as we know, only DNMT1, DNMT3a, and DNMT3b interplay to produce the global cytosine methylation pattern. These independently encoded proteins are classified as de novo enzymes (DNMT3a and DNM3b) or as a maintenance enzyme (DNMT1). DNMT2 and DNMT3L are not thought to function as cytosine methyltransferases. However, DNMT3L was shown to stimulate de novo DNA methylation by DNMT3a and to mediate transcriptional repression through interaction with histone deacetylases $1 .{ }^{8,9}$

Abnormal patterns of DNA methylation in cancer cells have been recognized for over 30 years. In particular, an abnormal gain of DNA methylation, hypermethylation, is now established as a very common event in cancer cells which often involves normally unmethylated gene promoter $\mathrm{CpG}$ islands ${ }^{5}$ (Fig. 1B). This promoter change can be associated with transcriptional silencing, and thus loss of function, of tumor suppressor genes and may be a key event contributing to the oncogenic process. ${ }^{10,11}$ The tumor suppressors that are inactivated by hypermethylation can affect DNA repair, programmed cell death, angiogenesis, cell cycle regulation, and tumor cell invasion. For example, the mismatch repair gene $h M L H 1$ is frequently silenced by methylation and results in mismatch repair deficiency in cancers of the colon, stomach, and endometrium. ${ }^{12,13}$ Other well-known examples of genes silenced by hypermethylation include the VHL tumor suppressor in renal cell carcinoma, ${ }^{14}$ the BRCAI tumor suppressor, ${ }^{15}$ the cyclin-dependent kinase inhibitor $C D K N 2 A(p 16),{ }^{16}$ and the retinoblastoma susceptibility gene $R B 1 .{ }^{17}$ Recently, several hundred genes silenced by DNA 
hypermethylation in various cancers have been identified using genome-wide approaches. ${ }^{18,19}$

\section{DNA METHYLATION AND CANCER}

Aberrant DNA methylation was the first epigenetic hallmark to be associated with cancer as a consequence of the alteration it causes in normal gene regulation. ${ }^{20}$ These alterations are of three types: hypermethylation, hypomethylation, and loss of imprinting. DNA hypermethylation refers principally to the gain of methylation at specific sites that are unmethylated under normal conditions. This aberrant methylation occurs mainly in promoter $\mathrm{CpG}$ islands, which are considered to be DNA sequences ( $>200$-bp window) with a GC content greater than $50 \%{ }^{21}$ This phenomenon of aberrant promoter $\mathrm{CpG}$ island hypermethylation has been associated with the stabilization of transcriptional repression and loss of gene function, and occurs fundamentally in tumor suppressor genes (Table 1). ${ }^{7,22}$

DNA hypomethylation is associated mainly with the loss of DNA methylation in genome-wide regions, although it can also occur locally. Feinberg and Vogelstein were the first to report that substantially more hypomethylation was found in the genes of cancer cells compared with their normal counterparts in two distinct histological types of cancer. ${ }^{20}$ DNA hypomethylation occurs in many gene-poor genomic areas, including repetitive elements, retrotransposons, and introns, where it leads to genomic instability. ${ }^{7}$ In repeat sequences, this is achieved by a higher rate of chromosomal rearrangements and, in retrotransposons, by a higher probability of translocation to other genomic regions. ${ }^{23,24}$ During tumor progression, the degree of hypomethylation of genomic DNA increases as the lesion derives from a benign proliferation of cells to an invasive cancer. ${ }^{25}$ Example of these exceptions are the fully methylated CpG islands associated with many transcriptionally silent genes on the $\mathrm{X}$ chromosome of females and the silenced alleles of some "imprinted genes", which through parental determination are programmed such that only one allele of the gene is expressed in normal tissues. ${ }^{2,26}$ Loss of imprinting is defined as the loss of the parental allele specific monoallelic expression of genes due to aberrant hypomethylation profiles at one of the two parental alleles. For example, loss of imprinting of IGF2 has been associated with an increased risk of cancer, including colorectal cancer. This event has been observed in different types of neoplasia. ${ }^{27} \mathrm{~A}$ number of studies have described DNA hypomethylation in several tumor types, such as colorectal and gastric cancers, melanomas, and others. ${ }^{28}$
Table 1. Representative Tumor Suppressor Genes Silenced by CytosinePhospho-Guanine (CpG) Island Promoter Hypermethylation in Various Cancer Types

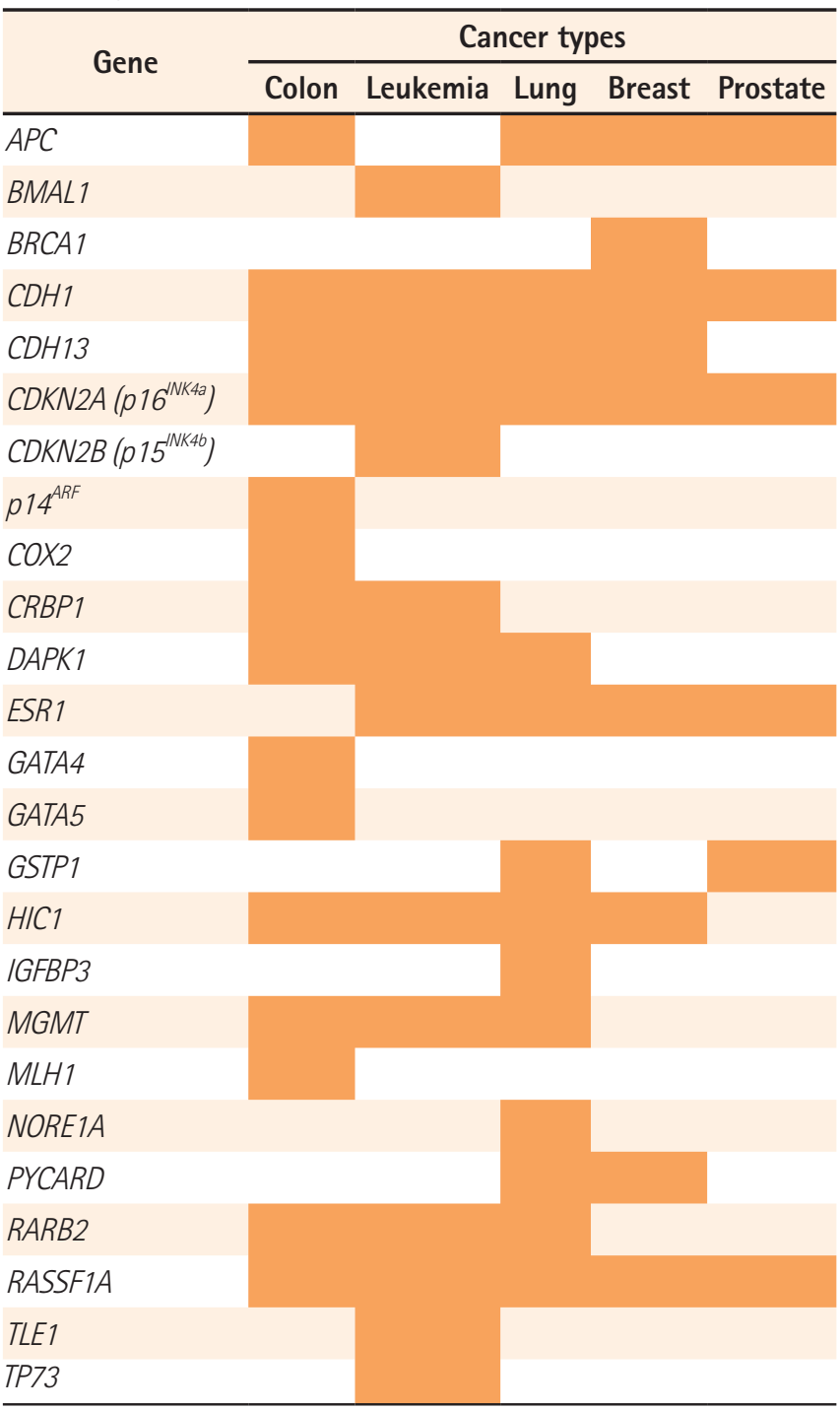

$A P C$, adenomatous polyposis coli; $B M A L 1$, brain and muscle arntlike protein 1 ; $B R C A 1$, breast cancer 1 , early onset; $C D H 1$, cadherin 1; $C D H 13$, cadherin $13 ; C D K N 2 A\left(p 16^{I N 4 a}\right)$, cyclin-dependent kinase inhibitor $2 A ; C D K N 2 B\left(p 15^{I N K 4 b}\right)$, cyclin-dependent kinase inhibitor $2 \mathrm{~B} ;$ p $^{A R F}$, cyclin-dependent kinase inhibitor $2 \mathrm{~A} ;$ COX2, cytochrome c oxidase subunit $2 ; C R B P 1$, cellular retinol binding protein 1; DAPK1, death-associated protein kinase 1; ESR1, estrogen receptor 1; GATA4, GATA binding protein 4; GATA5, GATA binding protein 5; GSTP1, glutathione $\mathrm{S}$-transferase pi $1 ; \mathrm{HIC1}$, hypermethylated in cancer 1 ; IGFBP3, insulin-like growth factor binding protein 3; MGMT, $0^{6}$ methylguanine-DNA methyltransferase; $M L H 1$, MutL homolog 1; NORE1A, Ras association (RalGDS/AF-6) domain family member 5; $P Y C A R D, P Y D$ and CARD domain containing; RARB2, retinoic acid receptor b2; RASSF1A, ras association (RaIGDS/AF-6) domain family $1 A ; T L E 1$, transducin-like enhancer of split 1, homolog of drosophila $E$ (sp1); TP73, tumor protein p73. 


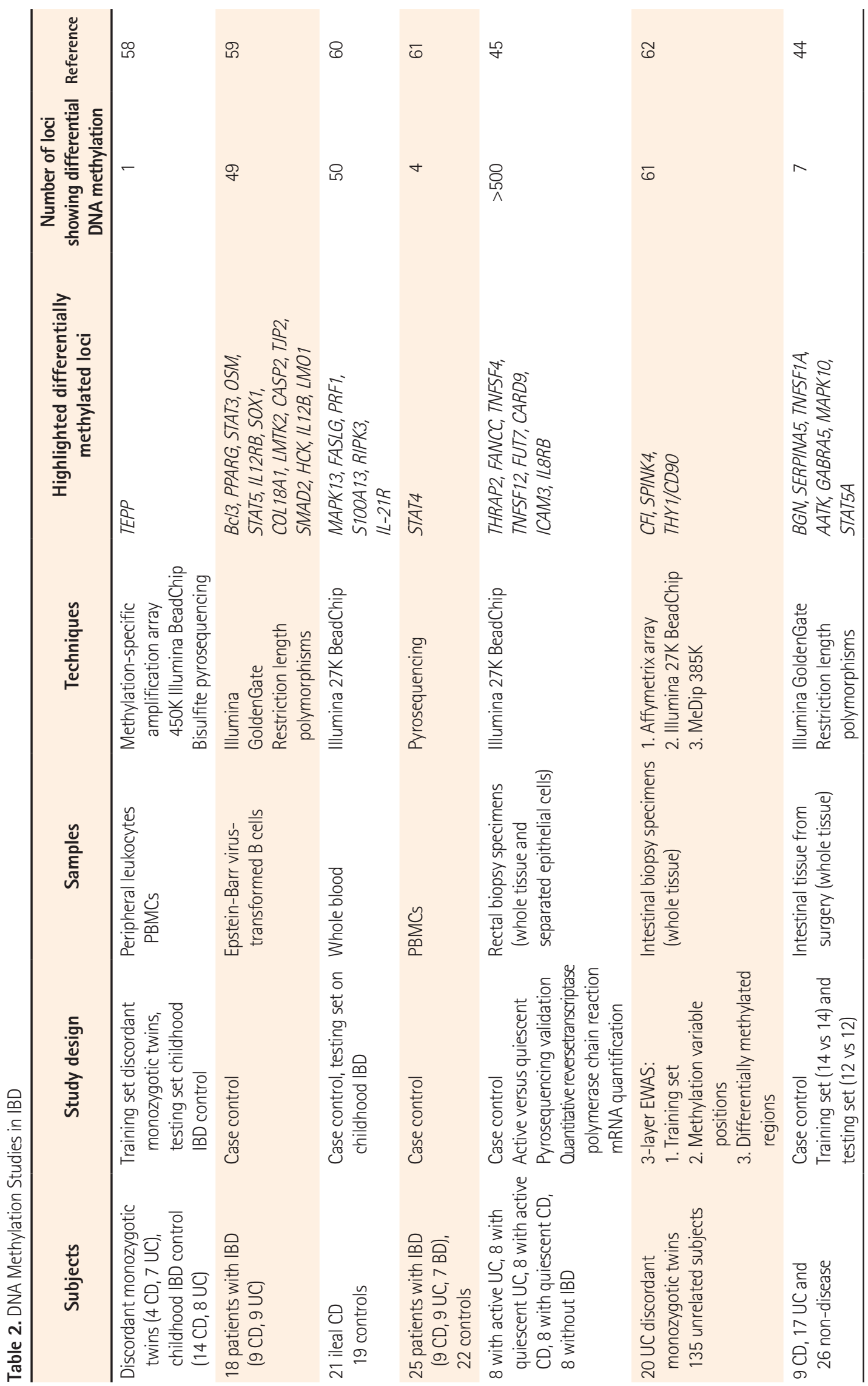




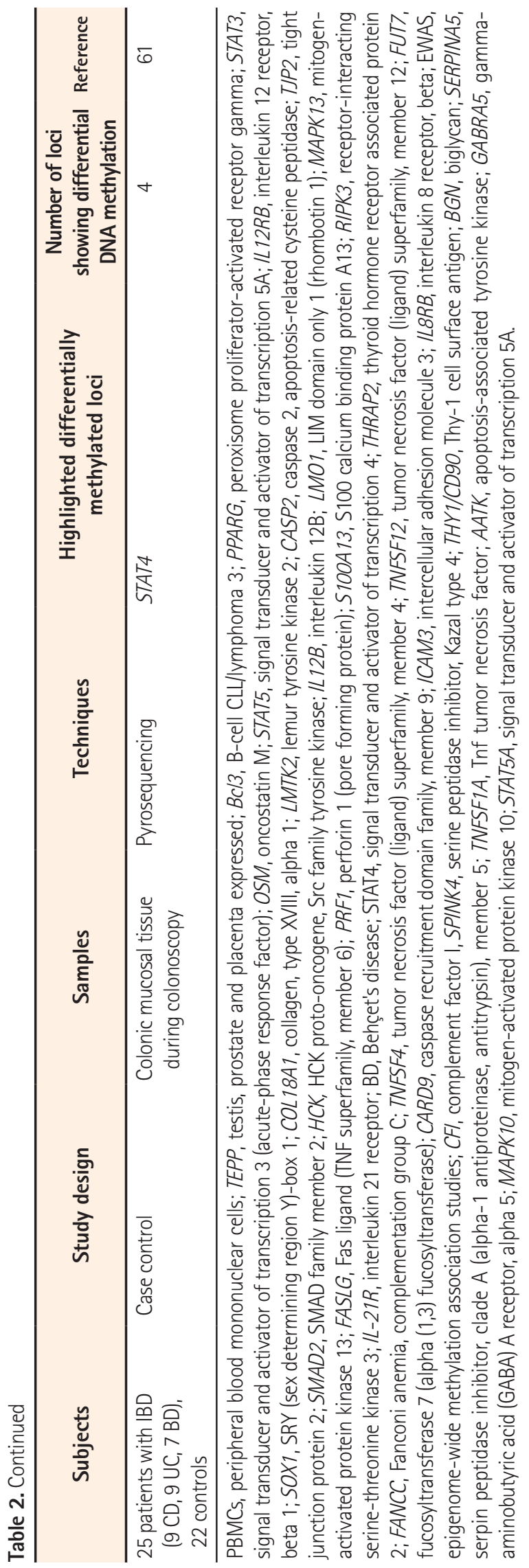

\section{DNA METHYLATION IN UC}

IBD, encompassing CD and UC, are chronic, relapsing, remitting, or continuously active diseases of the gastrointestinal tract and are occasionally associated with extra-intestinal manifestations. ${ }^{29}$ Unlike in Europe and the US, during the last two decades, IBD has been very rare in Asia. ${ }^{30}$ Although recent population-based and referral center cohorts have shown a rising incidence and prevalence of IBD in Asia. ${ }^{31}$

Although the exact etiology of IBD remains unknown, current research focuses on dysregulation of the immune response against the intestinal flora in genetically susceptible individuals. ${ }^{29,32}$ The pathogenesis of IBD is believed to involve an aberrant immune response to intestinal microbiota in genetically susceptible individuals. ${ }^{33,34}$ Genetic studies have provided many candidate loci in the past decade, and the innate and acquired immune responses have been implicated in pathogenesis. ${ }^{33}$ However, identified genetic factors account for only a modest proportion of the disease variance: $13.6 \%$ for CD and $7.5 \%$ for UC. ${ }^{35}$ Overall, all known genetic risk factors can only account for approximately $20 \%$ of the genetic risk. ${ }^{36,37}$

More specifically, IBD could be caused by interactions between the host and the environment, which encompass the intestinal microbiota, the immune system, the genetic composition of the host, and specific environmental factors such as the effects of smoking, breastfeeding, drugs, dietary products, and so on ${ }^{29,34,35}$ Regarding the interaction between environment and genome, epigenetic mechanisms and more specifically DNA methylation seem to be of great importance. $^{38}$

UC represents one major sub-phenotype of human IBD. In past decades, UC has displayed a remarkably steep rise in incidence, which cannot be explained by genetic variants alone. Beyond germ line DNA variants, epigenetic variants, e.g., DNA methylation and histone modifications, could modulate disease-relevant gene function. ${ }^{39}$ Methylation studies extend to different kinds of genes and genetic loci, trying to detect the possible correlation of their methylation to IBD. Indeed, epigenetic modifications represent promising candidates for elucidating processes of disease manifestation beyond the identified risk loci based on recent literature (Table 2).

There are several reports that the hypermethylation of many gene promoters is associated with UC patients. Firstly, Gloria et al..$^{40}$ has reported that DNA methylation relates to UC pathogenesis, which found that incorporation of the $3^{\mathrm{H}}$ methyl group into DNA was 10-fold higher in UC patients 
UC (Kim et al., 2013)
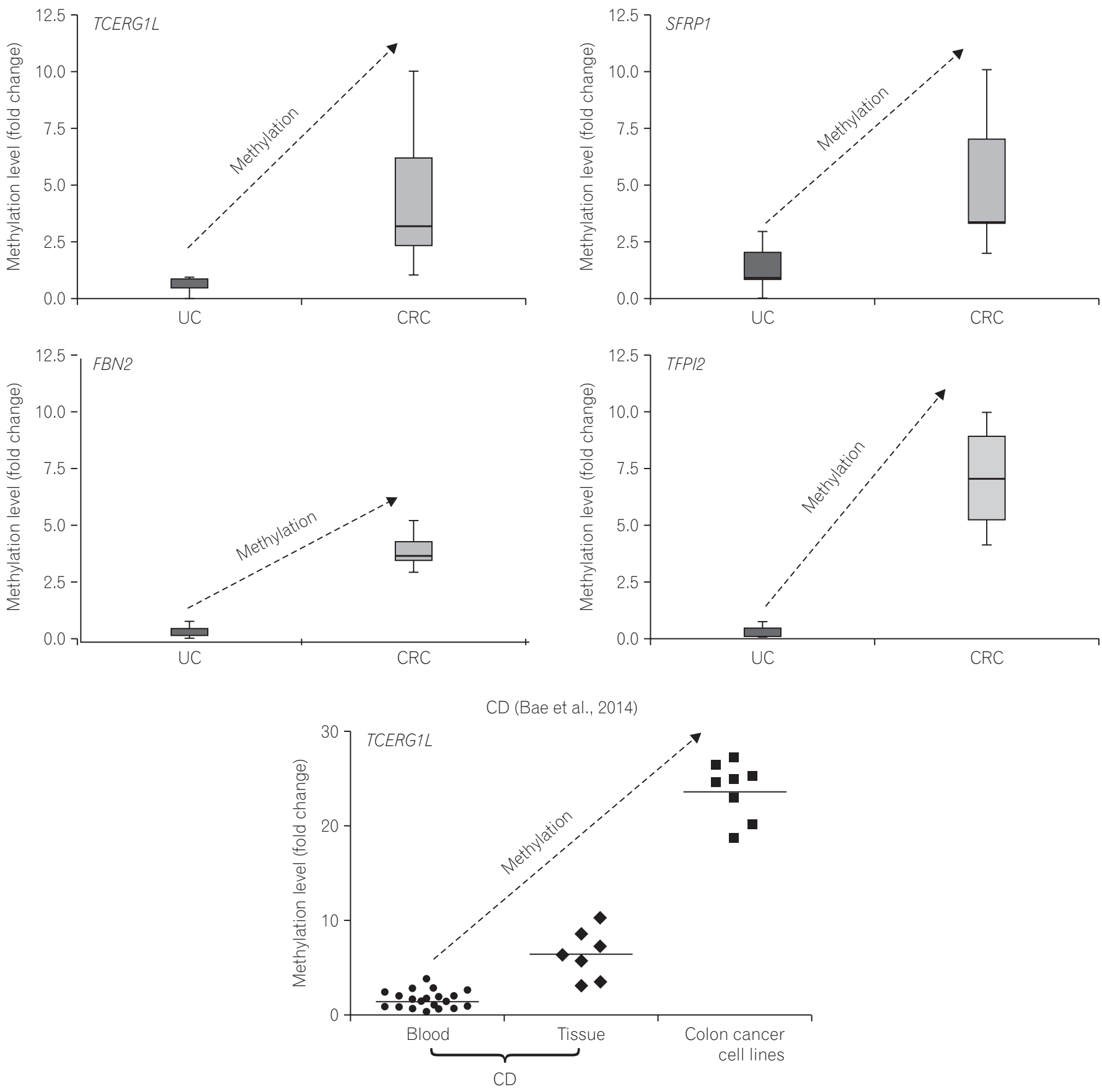

Fig. 2. Methylation level of candidate genes in IBD patients. DNA methylation of several candidate genes has been detected in UC tissues, CD tissues, and CD patient blood samples. The graph was quoted and modified from our previous publications (UC data from Kim et al. ${ }^{43} 2013$ and CD data from Bae et al., ${ }^{47}$ 2014). CRC, colorectal cancer; TCERG1L, transcription elongation regulator 1-like; SFRP1, secreted frizzled-related protein 1; FBN2, fibrillin 2; TFPI2, tissue factor pathway inhibitor 2.

than in controls, and significantly higher in histologically active than in inactive disease. Other studies on many kinds of genes, such as E-cadherin (cell-cell adhesion molecule), p16 (P16INK4a), CDH1, GDNF, and MDR1, proved that their promoter methylation was detected in high frequencies in UC patients. The most interesting gene here is $\mathrm{CDHI}$ encoding E-cadherin, which plays a central role in epithelial cell-cell adhesion. Since $C D H 1$ has been reported to be 
down-regulated in areas of UC inflammation, there is growing evidence of DNA methylation of $\mathrm{CDH} 1$ in IBD disease. Therefore, promoter hypermethylation of this gene has confirmed that it is associated with long standing inflammation, and that fact that the DNA methylation of this gene may be implicated in UC may make it a useful biomarker for detecting patients at high risk for developing colorectal cancer., ${ }^{41,42}$ Very recently, we reported that we had newly identified a number of genes (SFRP1, TFPI2, TCERGIL, and FBN2) that are highly methylated in colon cancer patients using a genome-wide approach in Korean UC patients ${ }^{43}$ (Fig. 2). Taken together, DNA methylation appears to be emerging as a common phenomenon in UC, especially in mucosal biopsies from inflamed tissue. Moreover, DNA methylation has been related to many different clinical aspects, such as disease severity, disease duration, disease phenotype, disease extent, and active inflammation and dysplasia.

\section{DNA METHYLATION IN CD}

Several reports have suggested that there are significant differential DNA methylation statuses between normal and inflamed tissues from CD and UC patients. ${ }^{44-46}$ One of the most comprehensive genome wide studies attempted to elucidate the molecular basis of CD disease, and identified a number of distinct susceptibility loci conclusively associated with CD pathogenesis. ${ }^{37}$ Although, unlike in UC, limited data have been reported regarding the contribution of DNA methylation status to CD pathogenesis, very recently we reported that we could detect DNA promoter hypermethylation in CD patients' serum using the cancer specific and highly frequency methylated gene TCERG1L $L^{47}$ (Fig. 2). Our data strongly suggest that DNA methylation could be a valuable molecular tool for screen CD or UC patients. However, more specific genes or loci should be identified, and comprehensive DNA methylation genome-wide studies should be conducted to further the understanding the contribution of DNA methylation in CD.

\section{DNA METHYLATION BIOMARKERS FOR CLINICAL USE}

Despite our constantly growing understanding of carcinogenesis, there is still an urgent need to design novel, powerful tools that can be applied in clinical practice. Cancer biomarkers are indispensable not only for early diagnosis, but also for improving prognoses, the prediction of therapeutic response, monitoring therapy, or assessing the risk of recur- rence after curative surgery. Cancer-specific hypermethylation of $\mathrm{CpG}$ islands represents one of the most prevalent molecular changes in cancer cells, and detection of abnormal methylation has proven to be of great use in clinical practice.

The use of DNA markers has various advantages over proteins. Unlike proteins, DNA is stable, easy to isolate from different kinds of material, and relatively small amounts of material are needed to perform assays. This is due to several inherent advantages in strategies for the detection of hypermethylation. DNA is a relatively stable substance and can be obtained from a wide range of sources. It can be stored for long periods after collection from patients. DNA methylation is a widespread alteration throughout the cancer genome. This allows for the possibility of using assays to query many points in the genome and for combining their use in highly predictive models. Highly sensitive and specific technologies now exist that can query the methylation state of specific DNA locations using minimal amounts of nucleic acid that can be obtained from a wide array of clinical specimens. These techniques include methylation-specific PCR, highperformance liquid chromatography, mass spectrometrybased methylation detection (EpiTYPER), Methy-Light, pyrosequencing, and next generation sequencing. ${ }^{14,48,49}$ Assays like methylation-specific PCR and EpiTYPER are able to detect DNA methylation from even minute amounts of material such as urine and saliva, and are capable of utilizing DNA from both frozen and paraffin-embedded archived tissue. These advantages have enabled investigators to evaluate the diagnostic and prognostic utility of methylation of a large number of genes in a many tumor types. ${ }^{6}$ Some genetic alterations have already been proposed as being valuable in diagnosis, ${ }^{50}$ but it has also been suggested that changes in DNA methylation patterns may also aid in following cancer progression. $^{51}$

DNA methylation markers have been developed using targeted candidate gene approaches, as well as by systematic screening for markers using genome-scanning techniques. ${ }^{52}$ Therefore, DNA-methylation-based technologies have a promising future in both clinical diagnostics and therapeutics. DNA methylation markers have obvious applications in diagnostics, but can also contribute indirectly to therapeutics as predictors of therapeutic response. According to the review by Laird, ${ }^{52}$ DNA methylation patterns have proven to be most useful in the sensitive detection of disease, whereas profiling methods are useful for stratification approaches.

With these genome-wide techniques for their identification and subsequent functional analyses, the number of 
potential DNA methylation biomarker genes available and the knowledge of their roles in cancer are rapidly increasing. One of the most important criteria for clinically useful biomarkers is whether they are applicable to surrogate tissues such as blood or other fluids that can be obtained through minimally invasive procedures. The sensitive and specific detection of tumor-specific DNA methylation patterns at distal sites makes DNA methylation a great candidate biomarker for early the detection of cancer and the clinical management of cancer patients. ${ }^{53,54}$ DNA methylation can be used as a molecular prognosis biomarker of potentially curable, stage I non-small-cell lung cancer, ${ }^{55}$ for which several methylated genes allow the identification of high risk patients who require special adjuvant therapies. In colon cancer, methylation of IGFBP3 and $E V L$ genes, which are identified from genome-wide techniques, predicted poor outcomes in colon cancer patients. ${ }^{56}$

DNA methylation biomarkers can also predict responses to chemotherapy. The best example is promoter hypermethylation of $O^{6}$-methylguanine-DNA methyltrasferase (MGMT), a DNA repair enzyme, in glioma. MGMT hypermethylation was detected in $40 \%$ of glioma and colorectal cancers in a study of a huge primary tumor cohort. ${ }^{57}$

\section{CONCLUSIONS}

Epigenetic studies carry significant discovery potential and may provide new insights into our understanding of the pathogenesis of IBD. Several examples of DNA methylation of specific genes or loci could be key factors in the translational study of IBD. Recent advances in our knowledge of IBD-associated DNA methylation underlie many promising clinical applications such as molecular biomarkers for diagnosis and prognosis as well as prediction of treatment outcomes. Even if the results from different studies are encouraging, limitations have thus far prevented widespread clinical application. One possible reason could be that DNA methylation frequency of many candidate genes is not high enough to achieve the sensitivity needed for clinical use. Various experiments new to the field of IBD research can be applied to the detection of IBD. Discovery of epigenetic changes in CD and UC patients may lead to the development of new therapies for IBD. In addition, technical advances in the near future are expected to reduce these problems, and DNA methylation is expected to play a key role in the development of personalized medicine.

\section{REFERENCES}

1. Kinzler KW, Vogelstein B. Lessons from hereditary colorectal cancer. Cell 1996;87:159-170.

2. Bird A. DNA methylation patterns and epigenetic memory. Genes Dev 2002;16:6-21.

3. Russo VEA, Martienssen RA, Riggs AD. Epigenetic mechanisms of gene regulation. New York: Cold Spring Harbor Laboratory Press, 1996.

4. Jones PA, Laird PW. Cancer epigenetics comes of age. Nat Genet 1999;21:163-167.

5. Herman JG, Baylin SB. Gene silencing in cancer in association with promoter hypermethylation. N Engl J Med 2003;349:20422054

6. Esteller M. Cancer epigenomics: DNA methylomes and histone-modification maps. Nat Rev Genet 2007;8:286-298.

7. Esteller M. Epigenetics in cancer. N Engl J Med 2008;358:11481159.

8. Chedin F, Lieber MR, Hsieh CL. The DNA methyltransferaselike protein DNMT3L stimulates de novo methylation by Dnmt3a. Proc Natl Acad Sci U S A 2002;99:16916-16921.

9. Deplus R, Brenner C, Burgers WA, et al. Dnmt3L is a transcriptional repressor that recruits histone deacetylase. Nucleic Acids Res 2002;30:3831-3838.

10. Jones PA, Baylin SB. The epigenomics of cancer. Cell 2007;128:683-692.

11. Wajed SA, Laird PW, DeMeester TR. DNA methylation: an alternative pathway to cancer. Ann Surg 2001;234:10-20.

12. Gu M, Kim D, Bae Y, Choi J, Kim S, Song S. Analysis of microsatellite instability, protein expression and methylation status of hMLH1 and hMSH2 genes in gastric carcinomas. Hepatogastroenterology 2009;56:899-904.

13. Kane MF, Loda M, Gaida GM, et al. Methylation of the $h M L H 1$ promoter correlates with lack of expression of hMLH1 in sporadic colon tumors and mismatch repair-defective human tumor cell lines. Cancer Res 1997;57:808-811.

14. Herman JG, Graff JR, Myohanen S, Nelkin BD, Baylin SB. Methylation-specific PCR: a novel PCR assay for methylation status of CpG islands. Proc Natl Acad Sci U S A 1996;93:9821-9826.

15. Esteller M, Silva JM, Dominguez G, et al. Promoter hypermethylation and BRCAl inactivation in sporadic breast and ovarian tumors. J Natl Cancer Inst 2000;92:564-569.

16. Merlo A, Herman JG, Mao L, et al. 5' CpG island methylation is associated with transcriptional silencing of the tumour suppressor p16/CDKN2/MTS1 in human cancers. Nat Med 1995;1:686692. 
17. Greger V, Passarge E, Hopping W, Messmer E, Horsthemke B. Epigenetic changes may contribute to the formation and spontaneous regression of retinoblastoma. Hum Genet 1989;83:155158.

18. Chan TA, Glockner S, Yi JM, et al. Convergence of mutation and epigenetic alterations identifies common genes in cancer that predict for poor prognosis. PLoS Med doi: 10.1371/journal. pmed.0050114. Published online 27 May 2008.

19. Schuebel KE, Chen W, Cope L, et al. Comparing the DNA hypermethylome with gene mutations in human colorectal cancer. PLoS Genet 2007;3:1709-1723.

20. Feinberg AP, Vogelstein B. Hypomethylation distinguishes genes of some human cancers from their normal counterparts. Nature 1983;301:89-92.

21. Takai D, Jones PA. Comprehensive analysis of CpG islands in human chromosomes 21 and 22. Proc Natl Acad Sci U S A 2002;99:3740-3745.

22. Bird A. The essentials of DNA methylation. Cell 1992;70:5-8.

23. Eden A, Gaudet F, Waghmare A, Jaenisch R. Chromosomal instability and tumors promoted by DNA hypomethylation. Science 2003;300:455.

24. Rodriguez J, Frigola J, Vendrell E, et al. Chromosomal instability correlates with genome-wide DNA demethylation in human primary colorectal cancers. Cancer Res 2006;66:8462-9468.

25. Fraga MF, Herranz M, Espada J, et al. A mouse skin multistage carcinogenesis model reflects the aberrant DNA methylation patterns of human tumors. Cancer Res 2004;64:5527-5534.

26. Antequera F, Bird A. CpG islands. EXS 1993;64:169-185.

27. Lim DH, Maher ER. Genomic imprinting syndromes and cancer. Adv Genet 2010;70:145-175.

28. Kulis M, Esteller M. DNA methylation and cancer. Adv Genet 2010;70:27-56.

29. Kaser A, Zeissig S, Blumberg RS. Inflammatory bowel disease. Annu Rev Immunol 2010;28:573-621.

30. Loftus EV, Jr. Clinical epidemiology of inflammatory bowel disease: Incidence, prevalence, and environmental influences. Gastroenterology 2004;126:1504-1517.

31. Thia KT, Loftus EV, Jr., Sandborn WJ, Yang SK. An update on the epidemiology of inflammatory bowel disease in Asia. Am J Gastroenterol 2008;103:3167-3182.

32. Jones PA, Takai D. The role of DNA methylation in mammalian epigenetics. Science 2001;293:1068-1070.

33. Xavier RJ, Podolsky DK. Unravelling the pathogenesis of inflammatory bowel disease. Nature 2007;448:427-434

34. Kim JM. Antimicrobial proteins in intestine and inflammatory bowel diseases. Intest Res 2014;12:20-33.

35. Jostins L, Ripke S, Weersma RK, et al. Host-microbe interactions have shaped the genetic architecture of inflammatory bowel disease. Nature 2012;491:119-124.
36. Xavier RJ, Rioux JD. Genome-wide association studies: a new window into immune-mediated diseases. Nat Rev Immunol 2008;8:631-643.

37. Barrett JC, Hansoul S, Nicolae DL, et al. Genome-wide association defines more than 30 distinct susceptibility loci for Crohn's disease. Nat Genet 2008;40:955-962.

38. Bird AP, Wolffe AP. Methylation-induced repression-belts, braces, and chromatin. Cell 1999;99:451-454.

39. Petronis A. Epigenetics as a unifying principle in the aetiology of complex traits and diseases. Nature 2010;465:721-727.

40. Gloria L, Cravo M, Pinto A, et al. DNA hypomethylation and proliferative activity are increased in the rectal mucosa of patients with long-standing ulcerative colitis. Cancer 1996;78:2300-2306.

41. Hsieh CJ, Klump B, Holzmann K, Borchard F, Gregor M, Porschen R. Hypermethylation of the $\mathrm{p} 16^{\mathrm{INK} 4 \mathrm{a}}$ promoter in colectomy specimens of patients with long-standing and extensive ulcerative colitis. Cancer Res 1998;58:3942-3945.

42. Tahara T, Shibata T, Nakamura M, et al. Effect of MDR1 gene promoter methylation in patients with ulcerative colitis. Int J Mol Med 2009;23:521-527.

43. Kim TO, Park J, Kang MJ, et al. DNA hypermethylation of a selective gene panel as a risk marker for colon cancer in patients with ulcerative colitis. Int J Mol Med 2013;31:1255-1261.

44. Lin Z, Hegarty JP, Cappel JA, et al. Identification of diseaseassociated DNA methylation in intestinal tissues from patients with inflammatory bowel disease. Clin Genet 2011;80:59-67.

45. Cooke J, Zhang H, Greger L, et al. Mucosal genome-wide methylation changes in inflammatory bowel disease. Inflamm Bowel Dis 2012;18:2128-2137.

46. Saito S, Kato J, Hiraoka S, et al. DNA methylation of colon mucosa in ulcerative colitis patients: correlation with inflammatory status. Inflamm Bowel Dis 2011;17:1955-1965.

47. Bae JH, Park J, Yang KM, Kim TO, Yi JM. Detection of DNA hypermethylation in sera of patients with Crohn's disease. Mol Med Rep 2014;9:725-729.

48. Eads CA, Danenberg KD, Kawakami K, et al. MethyLight: a high-throughput assay to measure DNA methylation. Nucleic Acids Res 2000;28:e32.

49. Uhlmann K, Brinckmann A, Toliat MR, Ritter H, Nurnberg P. Evaluation of a potential epigenetic biomarker by quantitative methyl-single nucleotide polymorphism analysis. Electrophoresis 2002;23:4072-4079.

50. Chin L, Gray JW. Translating insights from the cancer genome into clinical practice. Nature 2008;452:553-563.

51. Caballero OL, Chen YT. Cancer/testis (CT) antigens: potential targets for immunotherapy. Cancer Sci 2009;100:2014-2021. 
52. Laird PW. The power and the promise of DNA methylation markers. Nat Rev Cancer 2003;3:253-266.

53. Glockner SC, Dhir M, Yi JM, et al. Methylation of TFPI2 in stool DNA: a potential novel biomarker for the detection of colorectal cancer. Cancer Res 2009;69:4691-4699.

54. Lofton-Day C, Model F, Devos T, et al. DNA methylation biomarkers for blood-based colorectal cancer screening. Clin Chem 2008;54:414-423.

55. Brock MV, Hooker CM, Ota-Machida E, et al. DNA methylation markers and early recurrence in stage I lung cancer. N Engl J Med 2008;358:1118-1128.

56. Yi JM, Dhir M, Van Neste L, et al. Genomic and epigenomic integration identifies a prognostic signature in colon cancer. Clin Cancer Res 2011;17:1535-1545.

57. Esteller M, Hamilton SR, Burger PC, Baylin SB, Herman JG. Inactivation of the DNA repair gene O6-methylguanine-DNA methyltransferase by promoter hypermethylation is a common event in primary human neoplasia. Cancer Res 1999;59:793797.
58. Harris RA, Nagy-Szakal D, Pedersen N, et al. Genome-wide peripheral blood leukocyte DNA methylation microarrays identified a single association with inflammatory bowel diseases. Inflamm Bowel Dis 2012;18:2334-2341.

59. Lin Z, Hegarty JP, Yu W, et al. Identification of disease-associated DNA methylation in B cells from Crohn's disease and ulcerative colitis patients. Dig Dis Sci 2012;57:3145-3153.

60. Nimmo ER, Prendergast JG, Aldhous MC, et al. Genome-wide methylation profiling in Crohn's disease identifies altered epigenetic regulation of key host defense mechanisms including the Th17 pathway. Inflamm Bowel Dis 2012;18:889-899.

61. Kim SW, Kim ES, Moon CM, Kim TI, Kim WH, Cheon JH. Abnormal genetic and epigenetic changes in signal transducer and activator of transcription 4 in the pathogenesis of inflammatory bowel diseases. Dig Dis Sci 2012;57:2600-2607.

62. Hasler R, Feng Z, Backdahl L, et al. A functional methylome map of ulcerative colitis. Genome Res 2012;22:2130-2137. 